\title{
Gunshot wounds to the spine: comparative analysis of two retrospective cohorts
}

Gonzalo Bertullo ${ }^{1}$, Hildo RC Azevedo-Filho ${ }^{2}$, Nivaldo S de Almeida ${ }^{3}$, Paulo Thadeu Brainer-Lima ${ }^{3}$, Renata Azevedo ${ }^{4}$, Fernanda Azevedo ${ }^{5}$

\begin{abstract}
The objective of this study is to compare two cohorts of patients affected by this disease in different periods and evaluate specifically the role of decompressed laminectomy. Analysis of 246 and 127 patients with gunshot wounds to the spine admitted to the Hospital da Restauração, Recife, Brazil, between January 1981 and June 1998, and January 2005 to December 2008. The general comparative features were: an experience of 17.5 and 3 years, $95.8 \%$ and $85.8 \%$ men and a mean age of 26.6 and 25 years. The neurologic condition at admission was Frankel/ASIA/IMSOP grade A (59.8\% and $38.6 \%)$; B (2.4\% and 30.7\%); C (20.8\% and 29.9\%); D (14.2\% and 0.8\%) and E (2.8\% and $0 \%$ ). Hospital stay mean were 28 and 30 days. Follow-up mean 76.3 days. Conservative management was three times higher than surgical treatment in period 2005-2008, 14.2\% and 12.6\% patients died respectively. We concluded that the decompressive laminectomies continued to be associated with a higher level of complications and did not improve patient's outcome.
\end{abstract}

Keywords: Gunshot wound; Gunshot wound to the spine; Spinal cord injury; Traumatic injury to the spine

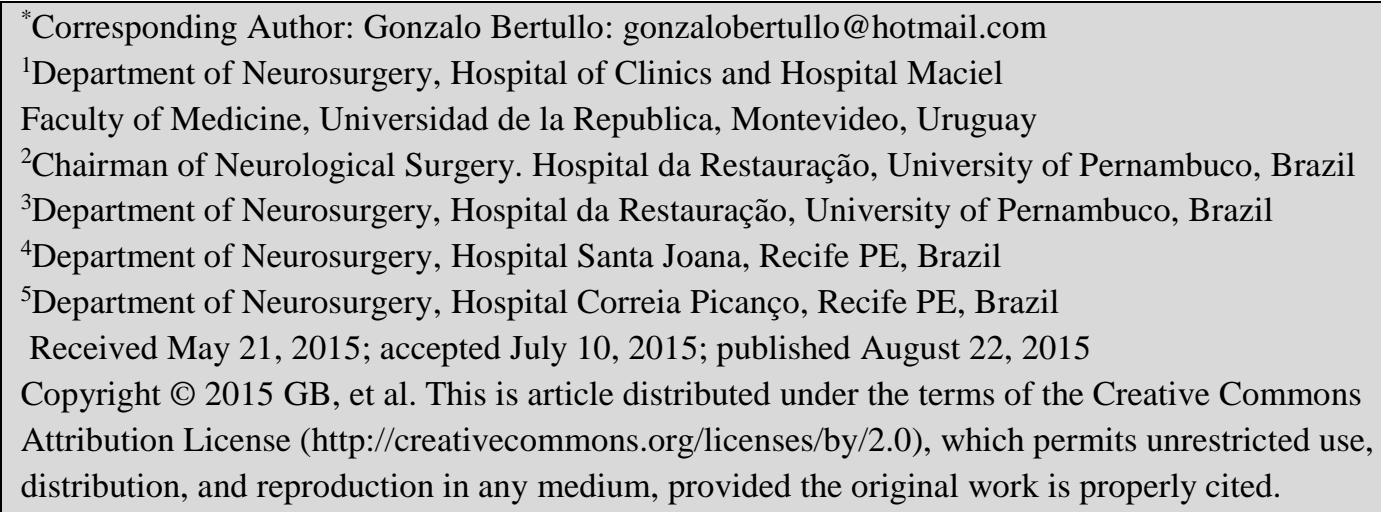

\section{Introduction}

Gunshot wounds to the spine (GWS) are the second most common cause of spinal cord injury (SCI) after vehicular trauma, accounting for $13 \%-17 \%$ of all cases of SCI but, at the same time, one of the less discussed [1]. While the incidence of SCI caused by vehicular trauma is 
decreasing, the incidence of SCI secondary to gunshot wounds (GW) continues to increase [2]. Few papers have been published on this topic and have considered in depth the effects of the available forms of treatment on the final outcome in the civilian population [3].

\section{Historical perspective}

Circa 1700 BC, Imhotep, in the Edwin Smith Papyrus, reported traumatic injuries to the spine (TIS) [4]. Galen (130-200 AD) was the first to show that longitudinal lesions to the cord did not cause severe functional damage, whereas transverse lesions were associated with paraplegia below the lesion. When the lesion was made between the first and the fourth cervical segments, all movements and sensations below the incision level disappeared. He also observed that lesions between the fourth cervical and the second thoracic segments were accompanied by less diaphragmatic and respiratory muscle disturbance, and this was progressively more evident when the lesion was made at even lower levels. Because of these observations, Galen could be considered the pioneer of spinal surgery [5]. Paul of Aeginet (625-690 AD) was one of the first authors to recommend decompressive laminectomy (DL) as the treatment for acute spinal compressive lesions [6]. Ambroise Paré (1557) established the first description of a SCI caused by a missile weapon [7]. Geraud (1753), during a session of the Acadèmie Royale de Chirurgie, reported a case where the bullet, entrapped in the body in the third lumbar vertebra, caused bladder and lower limb paralysis. He eventually removed the bullet. Although the wound became infected, the patient obtained a slight recovery of muscle power in the lower limbs [7]. Admiral Nelson was victim of this kind of lesion. During the final hours of the Battle of Trafalgar, he was fatally wounded by a bullet that entered through the thoracic cavity and reached the spinal cord, causing immediate paraplegia. His surgeon said, "My lord, unfortunately for our country, there is nothing that can be done." Lord Nelson died shortly after that [4]. James A. Garfield, the 20th president of the United States, also suffered a GWS.

There was an incomplete conus and cauda equina lesion, leading to his death 79 days later [4]. According to Sonntag [35] during the American Civil War, 642 cases of GWS were reported in the Medical and Surgical History of the War of the Rebellion. Surgical options were not discussed, probably because of the 
lack of surgical resources at that time. The death rate reached $55 \%$. It is ironic to think that increasing spinal cord trauma is the result of the same technological advances that later allowed its study. The incidence of TWS had grown side by side with the Industrial Revolution and the invention of cars and modern weapons [4]. The introduction of x-ray imaging allowed anatomic understanding of GW lesions [8]. Harvey Cushing, who began studying the application of $\mathrm{x}$-rays to the investigation of neurologic problems, pioneered its intraoperative use. Cushing's first publication dealt with the role of $\mathrm{x}$-rays in the diagnostic workup of a neurosurgical patient [9]. In 1896 he admitted a woman who had been shot by her husband; she presented with BrownSequard syndrome, and the $\mathrm{x}$-ray disclosed a bullet lodged in the lower cervical SC [10]. During World War I, Cushing developed the basis for modern techniques for the treatment of gunshot injuries to the nervous system. Despite his efforts, the results in cord wounds were disappointing. Only patients with incomplete lesions of the cord survived, and the overall death rate exceeded $60 \%$, probably as a result of the more powerful weapons then in use $[9,7]$. World War II was marked by changes in surgical techniques and the use of antibiotics. The management of SCI at the end of the conflict consisted of early surgery, with removal of debris and the application of dural patches when needed. The death rate decreased to $14.1 \%$ among 485 patients [11]. Spurling when studying 1,260 patients, observed $15 \%$ functional recovery, mainly patients with incomplete lesions. British authors also reported a positive change. Guttmann [12] reported a death rate of $9.3 \%$. This improvement was attributed to the standardized postoperative management of patients with SCI, including prevention of decubiti and urinary and respiratory infections and the use of blood and blood products. More recent conflicts such as Israel and Lebanon, Iran and Iraq and the Balkans war have shown that surgery in itself was not responsible for the lower death rate. Indeed, surgical management was associated with higher rates of complications that reached $14.5 \%$ and included cerebrospinal fluid (CSF) fistulas, meningitis, and wound infection $[13,14,15,16]$.

\section{Current data}

GW in civilians occur with shorter guns with lower velocities and smaller calibers [17]. According to Miller guns with calibers $22,25,32$, and 38 are the 
most frequently used, and the patients wounded in this setting are mainly young men [18, 19]. In the United States, African Americans are involved in $53 \%$ of the events, Latinos in $28 \%$, whites in 18\%, and Asians in $1 \%$, probably reflecting the variety of social, economic, and cultural issues that exist in urban centers [20]. GW to the spine in civilians most frequently involve the thoracic region $(54 \%)$; the lumbosacral area and the cervical area are affected in $33 \%$ and $13 \%$, respectively [21]. The experience acquired treating war lesions predicted that the surgical indications and options would be used to treat civilian injuries [22, 20]. However, recent studies have pointed out that civilian lesions can differ significantly from injuries sustained on the battlefield [23]. Simpson et al., [20] in a retrospective study, showed no benefit from DL in civilian patients; indeed, a higher rate of complications was seen in the surgically treated group. Kupcha et al., analyzing 28 patients with $\mathrm{GW}$ to the cervical region, observed a very similar result [24]. No clear benefit was seen in patients with complete or incomplete lesions who underwent surgical decompression. Infection and instability occurred most frequently in this group. The presence of a bullet inside the spinal canal, or its removal, made no difference in the final outcome of patients with complete lesions. Although recognizing the small number of publications that address this matter, Yashon et al., Benzel et al., and Miller recommended that functional deterioration, incomplete lesions, and the presence of bone or metallic fragments in the spine are indications for DL $[25,26,18]$. Heiden et al., suggested surgical treatment as a way to remove debris and correct CSF leak [27]. Turgut et al., observing 17 patients between 1968 and 1990, found that DL was an important event for neurologic improvement [26]. Yoshida et al., described a significant association between removal of a bullet inside the thoracolumbar canal and neurologic improvement [28].

\section{Patients and Method}

Two retrospective cohorts of 246 [29] and 127 patients with GWS, assisted in the Department of Neurosurgery, Hospital da Restauração / Recife, Brazil, between January 1981 to June 1998 and January 2005 to December 2008 respectively were studied.

All those SCI caused by firearms for civilian use were included. We took a data collection sheet and a database with the following variables: age, sex, and 
neurological condition at admission, length of hospital stay and follow-up, spinal injury level and intra-axial, radiologic findings, therapeutic management, laminectomy and surgical opportunity, infectious complications, CSF leak post-surgery, deaths and outcome. Frankel / ASIA / IMSOP was used to classify and evaluate the neurological condition at admission and evolution [3]. The change in at least one grade was considered to establish improvement or deterioration at the same. All patients received active physiotherapy during their hospital stay. Vertebral injury level was classified as cervical or thoracolumbar. Cervical instability was evaluated using neutral and dynamic x-ray according to the recommendations Bailey and White et al., and the thoracolumbar area following to Denis.

Intra-axial injury was subdivided as spinal cord or cauda equina $[30,31,32]$ Metallic fragments or bullet inside canal were radiological findings searched. A bullet was considered lodged in the spinal canal when $50 \%$ or more of its mass was inside the canal. Therapeutic management was considered surgical or conservative and the opportunity was defined as an emergency if surgery was performed within the first 24 hours of admission or elective past 24 hours.

Laminectomy included all patients undergoing to posterior decompression through total removal of both laminae and yellow ligaments. Extensive dural lesions were repaired using fascia lata, and iliac bone crest was the donor site for all bone grafts used. Posterior cervical fixation was performed according to the Roy-Camille et al., Technique [33]. All patients undergoing cervical fixation wore an external orthesis (rigid collar) for 12 weeks. Respiratory infection and meningitis were the variables grouped into infectious complications. The outcome was defined in three items according to neurological condition: not unchanged, improved or worsened. Epidat and EPI INFO version 3.1 and 6.04 respectively were used for the description and analysis of the study population. The description of the qualitative data was performed as frequencies and percentages. Quantitative data were described as mean $(\chi)$ and standard deviation (SD). For bivariate analysis we used the Chi-square test with Yates correction or Fisher exact test when it was necessary, in the same way that the odds ratio $(\mathrm{OR})$ with confidence 
intervals at 95\% using Woolf method. Significance level was defined as $p$ value $<0.05$. This study was approved by the Hospital Ethics Committee.

\section{Results}

Two hundred forty-six patients were admitted in the first period which represented almost seventeen and a half, while a total of one hundred twentyseven patients in three years accounted for the second period. The comparative characteristics of sex, age, hospital stay and follow-up were similar (Table 1).

\begin{tabular}{ll}
$1981-1998$ Experience * & $2005-2008$ Experience \\
\hline $\mathrm{t}=17.5$ years & $\mathrm{t}=3$ years \\
$\mathrm{N}=246$ & $\mathrm{~N}=127$ \\
$235(95,5 \%)$ male & $109(85,8 \%)$ male \\
$\chi$ age $=26.6$ years (SD:9.2) & $\chi$ age $=25$ years (SD: 9.0$)$ \\
$\chi$ hospital day $=28$ days & $\chi$ hospital day $=30$ days \\
$\chi$ follow-up $=76.3$ days & $\chi$ follow-up $=76.3$ days \\
Death $=35(14,6 \%)$ & Death $=16(12,6 \%)$ \\
\hline${ }^{*}$ Cited $(29)$ &
\end{tabular}

\section{Table 1.}

General features

The neurological condition at admission in 2005-2008 showed a significant increase in patients classified as Frankel B previously considered A (Table 2).

\begin{tabular}{lll}
\hline & \multicolumn{2}{c}{ Patients } \\
\cline { 2 - 3 } Frankel/Asia/IMSOP & $1981-1998^{*}$ & $2005-2008$ \\
\hline A & $147(59,8 \%)$ & $49(38,6 \%)$ \\
B & $6(2,4 \%)$ & $39(30,7 \%)$ \\
C & $51(20,8 \%)$ & $38(29,9 \%)$ \\
D & $35(14,2 \%)$ & $1(0,8 \%)$ \\
E & $7(2,8 \%)$ & - \\
Total & $246(100 \%)$ & $127(100 \%)$ \\
\hline
\end{tabular}

Comparative Chi-square $=60.04, \mathrm{p}<0.0001$ *Cited (29)

Table 2.

Neurologic condition at admission

Thirty-five (14.2\%) patients died during the first study period (Table 3).

\begin{tabular}{lll}
\hline & \multicolumn{2}{c}{ Patients } \\
\cline { 2 - 3 } Deaths & $1981-1998^{*}$ & $2005-2008$ \\
\hline Yes & $35(14,2 \%)$ & $16(12,6 \%)$ \\
No & $211(85,8 \%)$ & $111(87,4 \%)$ \\
Total & $246(100 \%)$ & $127(100 \%)$ \\
\hline \multicolumn{2}{c}{ Comparative Chi-square $=0.188 ; \mathrm{p}=0.66$} \\
*Cited (29)
\end{tabular}

Table 3.

Deaths

The cervical lesion decreased significantly (29.3\% to $14.5 \%$ ) from one period to another. Thoracolumbar injuries were distributed in thoracic 113 (45.9\%), lumbar 61 (24.8\%) between 1981-1998 and thoracic 67 (52.8\%), lumbar 37 (29.1\%) in 2005-2008 (Table 4). In this last period there were three cases of Cervical-thoracic lesion $(2.4 \%)$ and two cases of thoracolumbar lesion $(1.6 \%)$. 


\section{Research Article}

\begin{tabular}{lcc}
\hline & \multicolumn{2}{c}{ Patients } \\
\cline { 2 - 3 } & $1981-1998 *$ & $2005-2008$ \\
\hline Cervical & $72(29,3 \%)$ & $18(14,5 \%)$ \\
Thoracolumbar & $174(70,7 \%)$ & $106(85,5 \%)$ \\
Total & $246(100 \%)$ & $124(100 \%)$ \\
\hline \multicolumn{2}{c}{ Comparative Chi-square $=9,75 ; \mathrm{p}=0.0018$} \\
*Cited (29)
\end{tabular}

\section{Table 4.}

Level of vertebral injury.

Pulmonary infection occurred in $48.6 \%$ and $44.4 \%$ of patients with cervical lesions more frequently than those with thoracic lesions $13.2 \%$ and $27.5 \%$ respectively. Cervical lesion showed six times more risk of pulmonary infection than thoracic lesions (Table 5).versus mild PE, normal versus severe PE and mild versus severe PE. Lastly regarding $\mathrm{Hb}$ level there was statically significant difference between normal versus severe PE.

\begin{tabular}{|c|c|c|c|c|c|c|}
\hline & \multicolumn{6}{|c|}{ Respiratory Infection } \\
\hline & \multirow[b]{2}{*}{ Yes } & \multicolumn{2}{|l|}{$1981-1998^{*(1)}$} & \multicolumn{3}{|c|}{$2005-2008^{(2)}$} \\
\hline & & No & Total & Yes & No & Total \\
\hline Cervical $^{(3)}$ & $35(48,6 \%)$ & $37(51,4 \%)$ & $72(100 \%)$ & $8(44,4 \%)$ & $10(55,6 \%)$ & $18(100 \%)$ \\
\hline Thoracolumbar & $23(13,2 \%)$ & $151(86,8 \%)$ & $174(100 \%)$ & $30(27,5 \%)$ & $79(72,5 \%)$ & $109(100 \%$ \\
\hline Total & $58(23,6 \%)$ & $188(76,4)$ & $246(100 \%)$ & $38(29,9 \%)$ & $89(70 \%)$ & $127(100 \%$ \\
\hline
\end{tabular}

Table 5.

Vertebral level versus respiratory infection.

The neurological condition was also associated with pulmonary infection, increasing the risk when Frankel is lower. There was no comparative significant difference (Table 6).

\begin{tabular}{|c|c|c|c|c|c|c|}
\hline \multirow{3}{*}{$\begin{array}{l}\text { Frankel-ASIA } \\
\text { IMSOP } \\
\text { Grade admission }\end{array}$} & \multicolumn{5}{|c|}{ Respiratory Infection } & \\
\hline & \multicolumn{3}{|c|}{$1981-1998 *(1)$} & \multicolumn{3}{|c|}{$2005-2008^{(2)}$} \\
\hline & Yes & No & Total & Yes & No & Total \\
\hline $\mathrm{A}+\mathrm{B}^{(3)}$ & $47(30.7 \%)$ & $106(69.3 \%)$ & $153(100 \%)$ & $30(37.0 \%)$ & $51(63.0 \%)$ & $81(100 \%)$ \\
\hline$C+D+E$ & $11(11.8 \%)$ & $82(88.2 \%)$ & $93(100 \%)$ & 08 (17.4\%) & $38(82.6 \%)$ & $46(100 \%)$ \\
\hline Total & $58(23.6 \%)$ & $188(76.4 \%)$ & $246(100 \%)$ & $38(29.9 \%)$ & $89(70.1 \%)$ & $127(100 \%)$ \\
\hline
\end{tabular}

Table 6.

Neurologic Condition versus Respiratory Infection.

During 1981-1998 respiratory infection was responsible for $41.4 \%$ of deaths with an eleven times increased risk in those subjects who were suffering. In the second period this fact declined significantly to $10.5 \%$ (Table 7).

\begin{tabular}{|c|c|c|c|c|c|c|}
\hline \multirow{3}{*}{$\begin{array}{l}\text { Respiratory } \\
\text { Infection }\end{array}$} & & \multicolumn{4}{|c|}{ Death } & \\
\hline & & \multicolumn{3}{|l|}{$1981-1998^{*(1)}$} & \multicolumn{2}{|l|}{$2005-2008^{(2)}$} \\
\hline & Yes & No & Total & Yes & No & Total \\
\hline$\overline{Y^{(3)}}{ }^{(3)}$ & $24(41.4 \%)$ & $34(58.6 \%)$ & $58(100 \%)$ & $4(10.5 \%)$ & $34(89.5 \%)$ & $38(100 \%)$ \\
\hline No & $11(5.9 \%)$ & 177 (94.1\%) & $188(100 \%)$ & $12(13.5 \%)$ & 77 (86.5\%) & $89(100 \%)$ \\
\hline Total & $35(14.2 \%)$ & $211(85.8 \%)$ & $246(100 \%)$ & $16(12.6 \%)$ & $111(87.4 \%)$ & $127(100 \%)$ \\
\hline
\end{tabular}

Table 7.

Respiratory infection versus death.

Table 8 shows the distribution according to treatment, the comparative highlight aspect is the significant increase of conservative management (63.8 to $84.1 \%$ ) and thus a decrease of surgeries (36.2\% to $20 \%$ ) mainly due to decrease in elective surgery (30.1 to $7.1 \%$ ). The comparative reason showed that operations were performed three times less in the second period (1.8 to 5.3) 
(Table 8). In the second period, patients admitted with favorable neurological condition (Frankel B $+\mathrm{C}+\mathrm{D}$ ) the election of conservative treatment was significantly higher, rising from $39.1 \%$ to $91 \%$ and therefore the surgery was the most likely option in patients with complete injury (Frankel A). From every two patients with incomplete injury who arrived in the period 1981-1998, one was operated (1.55:1), while in the second period was one in ten $(1: 10)$ (Table 9). We also noted a significant change in the surgical opportunity, complete injuries are most frequently emergency operated than incomplete in the first period (27.3 vs $10.7 \%$ ), however, emergency surgery in patients with incomplete injury in 2005 to 2008 (85.7 vs. $23.1 \%$ ) prevailed (Table 10).

\begin{tabular}{lrr}
\hline & \multicolumn{2}{c}{ Patients } \\
\cline { 2 - 3 } & \multicolumn{1}{c}{$1981-1998 *$} & $2005-2008$ \\
\hline Conservative & $157(63,8 \%)$ & $106(84,1 \%)$ \\
Elective surgery & $74(30,1 \%)$ & $9(7,1 \%)$ \\
Emergency surgery & $15(6,1 \%)$ & $11(8,7 \%)$ \\
Total & $246(100 \%)$ & $126(100 \%)$ \\
\hline \multicolumn{2}{c}{ Comparative Chi-square: $16.58 ; \mathrm{p}<0.0001$} \\
Statistical Reason C/S $1981-1998=1.8$ \\
Statistical Reason C/S 2005-2008=5.3 \\
*Cited (29)
\end{tabular}

Table 8.

Treatment.

\begin{tabular}{|c|c|c|c|c|c|c|}
\hline \multirow{3}{*}{$\begin{array}{l}\text { Frankel-ASIA } \\
\text { IMSOP } \\
\text { Grade admission }\end{array}$} & \multicolumn{6}{|c|}{ Surgery } \\
\hline & \multicolumn{3}{|c|}{$1981-1998^{*(1)}$} & \multicolumn{3}{|c|}{$2005-2008^{(2)}$} \\
\hline & $\overline{\text { Yes }}$ & No & Total & Yes & No & Total \\
\hline $\bar{A}$ & $33(22,4 \%)$ & $114(77,6 \%)$ & $147(100 \%)$ & $14(28,6 \%)$ & $35(71,4 \%)$ & $49(100 \%)$ \\
\hline $\mathrm{B}+\mathrm{C}+\mathrm{D}$ & $56(60,9 \%)$ & $36(39,1 \%)$ & $92(100 \%)$ & $07(9,0 \%)$ & $71(91 \%)$ & $78(100 \%)$ \\
\hline Total & $89(37,2 \%)$ & $150(62,8 \%)$ & $239(100 \%)$ & $21(16,5 \%)$ & $106(83,5 \%)$ & $127(100 \%)$ \\
\hline $\begin{array}{l}\text { (1) Chi-s } \\
\text { (2) Chi-s } \\
\text { (3) Comy } \\
\text { Statistica } \\
\text { Statistica } \\
\text { *Cited (29 }\end{array}$ & $\begin{array}{l}\text { quare }=35.74 \\
\text { quare }=8.37 \\
\text { parative }=48.7 \\
\text { Reason } B+C \\
\text { Reason } B+C \\
\text { ) }\end{array}$ & $\begin{array}{l}\mathrm{p}<0.0001 \\
\mathrm{p}=0.004 \\
4 ; \mathrm{p}<0.0001 \\
+\mathrm{D}=\text { surgery Yes } \\
+\mathrm{D}=\text { surgery } \mathrm{No} /\end{array}$ & $\begin{array}{l}\text { No } 1983-2000 \\
\text { Yes } 2005-200\end{array}$ & $\begin{array}{l}=1.55 \\
3=10.1\end{array}$ & & \\
\hline
\end{tabular}

Table 9.

Neurologic condition versus surgery

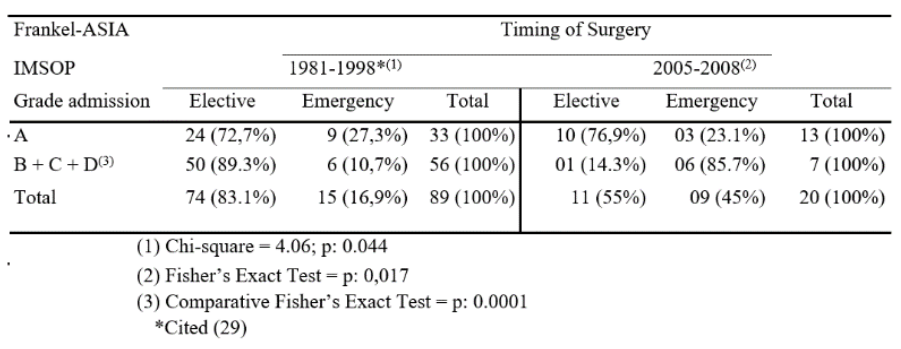

Table 10.

Neurologic Condition versus Timing of Surgery.

A greater mass of the intraspinal missile was significantly associated with the choice for surgical management during 1981-1998 (Table 11), although it was not statistically related to the need for emergency surgery (Table 12). There was no significance difference at the second period (62.5 vs $22.5 \%$ ) (Table 11 and 12).

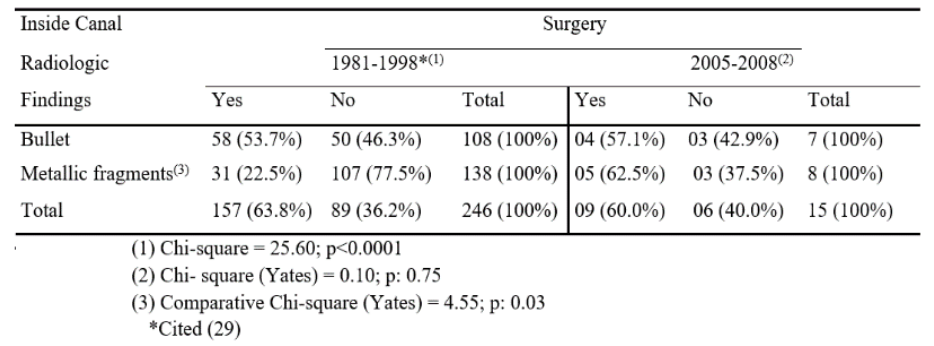

Table 11.

Radiologic findings versus surgery 


\begin{tabular}{lcrr|rrr}
\hline Inside Canal & \multicolumn{5}{c}{ Timing of Surgery } \\
\cline { 2 - 6 } Radiologic & \multicolumn{5}{c}{$1981-1998^{*(1)}$} \\
\cline { 2 - 6 } Findings & Elective & Emergency & \multicolumn{1}{c}{ Total } & Elective & Emergency & Total \\
\hline Bullet & $45(77.6 \%)$ & $13(22.4 \%)$ & $58(100 \%)$ & $04(57.1 \%)$ & $03(42.9 \%)$ & $7(100 \%)$ \\
Metallic fragments & $29(93.5 \%)$ & $2(6.5 \%)$ & $31(100 \%)$ & $05(62.5 \%)$ & $03(37.5 \%)$ & $8(100 \%)$ \\
Total & $74(83.1 \%)$ & $15(16.9 \%)$ & $89(100 \%)$ & $09(60.0 \%)$ & $06(40.0 \%)$ & $15(100 \%)$ \\
\hline \multicolumn{5}{c}{ (1) Fisher's Exact Test = p: 0.07} \\
& (2) Chi- square (Yates) $=0.10 ;$ p: 0.75 \\
*Cited (29)
\end{tabular}

Table 12.

Radiologic Findings versus Timing of Surgery

\begin{tabular}{|c|c|c|c|c|c|c|}
\hline \multirow[b]{3}{*}{ Lesion } & \multicolumn{6}{|c|}{ Surgery } \\
\hline & \multicolumn{3}{|c|}{$1981-1998^{*(1)}$} & \multicolumn{3}{|c|}{$2005-2008^{(2)}$} \\
\hline & Yes & No & Total & Yes & No & Total \\
\hline Spinal cord & $55(27.6 \%)$ & $144(72.4 \%)$ & $199(100 \%)$ & $11(14.3 \%)$ & $66(85.7 \%)$ & $77(100 \%)$ \\
\hline Cauda equina & $34(72.3 \%)$ & $13(27.7 \%)$ & $47(100 \%)$ & $10(21.3 \%)$ & $37(78.7 \%)$ & $47(100 \%)$ \\
\hline Total & $89(36.2 \%)$ & $157(63.8 \%)$ & $246(100 \%)$ & $21(16,9 \%)$ & $103(83,1 \%)$ & $124(100 \%)$ \\
\hline & $\begin{array}{l}\text { (1) Chi-squa } \\
\text { (2) Chi-squar } \\
\text { (3) Compara } \\
{ }^{*} \text { Cited ( } 29\end{array}$ & $\begin{array}{l}\mathrm{e}=32.9 ; \mathrm{P}<0 \\
\text { e: } 1,01 ; \mathrm{P}=0,3 \\
\text { ive Chi-square- }\end{array}$ & $\begin{array}{l}001 \\
4 . \\
24.61 \mathrm{p}<0.00\end{array}$ & & & \\
\hline
\end{tabular}

\section{Table 13.}

Lesion versus Surgery

A CSF leak was seen in $10(4.1 \%)$ patients during 1981-1998 and 12 (9.7\%) during 2005-2008, in both periods this complication was significantly associated with the surgical management and comparatively showed a significant increase in this complication at the second period going from 20 to $50 \%$ of operated patients $(2 / 10$ to $6 / 6)$. The results reflected that those operated patients have, approximately, seven times more risk of CSF leak (Table 14). Operated patients have four times more risk of developing meningitis during the second period, but not during the first, this comparative difference was significant (Table 15).
We observed an enormous impact upon finding that patients with postoperative CSF fistula were associated to 19 and 17 times more risk of suffer meningitis respectively (Table 16). Emergency surgery was associated statistically with meningitis only in the first study period (Table 17)

\begin{tabular}{|c|c|c|c|c|c|c|}
\hline \multirow[b]{3}{*}{ Surgery } & \multirow[b]{3}{*}{ Yes } & \multicolumn{4}{|c|}{ Leak } & \\
\hline & & \multicolumn{2}{|c|}{ 1981-1998*(1) } & \multicolumn{3}{|c|}{$2005-2008^{(2)}$} \\
\hline & & No & Total & Yes & No & Total \\
\hline$\overline{\text { Yes }}$ & $8(9.0 \%)$ & $81(91.0 \%)$ & $89(100 \%)$ & $06(28.6 \%)$ & $15(71.4 \%)$ & $21(100 \%)$ \\
\hline No & $2(1.3 \%)$ & $155(98.7 \%)$ & $157(100 \%)$ & $06(5.8 \%)$ & $97(94.2 \%)$ & $103(100 \%)$ \\
\hline Total & $10(4.1 \%)$ & $236(95.9 \%)$ & $246(100 \%)$ & $12(9.7 \%)$ & $112(90.3 \%)$ & $124(100 \%)$ \\
\hline
\end{tabular}

Table 14.

Surgery versus cerebrospinal fluid leak

\begin{tabular}{|c|c|c|c|c|c|c|}
\hline \multirow[b]{3}{*}{ Surgery } & \multicolumn{6}{|c|}{ Meningitis } \\
\hline & \multicolumn{3}{|c|}{$1981-1998 *(1)$} & \multicolumn{3}{|c|}{$2005-2008^{(2)}$} \\
\hline & Yes & No & Total & Yes & No & Total \\
\hline Yes & $8(9.0 \%)^{(3)}$ & $81(91.0 \%)$ & $89(100 \%)$ & $9(42.9 \%)$ & $12(57.1 \%)$ & $21(100 \%)$ \\
\hline No & $4(2.5 \%)$ & $153(97.5 \%)$ & $157(100 \%)$ & $17(16.0 \%)$ & $89(84.0 \%)$ & $106(100 \%)$ \\
\hline Total & $12(4.9 \%)$ & 234 (95.1\%) & $246(100 \%)$ & $26(20.5 \%)$ & $101(79.5 \%)$ & $127(100 \%)$ \\
\hline
\end{tabular}

Table 15.

Surgery versus meningitis

\begin{tabular}{|c|c|c|c|c|c|c|}
\hline \multirow[b]{3}{*}{ Leak } & \multicolumn{6}{|c|}{ Meningitis } \\
\hline & \multicolumn{3}{|c|}{$1981-1998 *(1)$} & \multicolumn{3}{|c|}{$2005-2008^{(2)}$} \\
\hline & Yes & No & Total & Yes & No & Total \\
\hline Yes & $4(40.0 \%)^{(3)}$ & $6(60.0 \%)$ & $10(100 \%)$ & $09(75.0 \%)$ & $03(25.0 \%)$ & $12(100 \%)$ \\
\hline No & $8(3.4 \%)$ & $228(96.6 \%)$ & $236(100 \%)$ & $17(15.2 \%)$ & $95(84.8 \%)$ & $112(100 \%)$ \\
\hline Total & $12(4.9 \%)$ & $234(95.1 \%)$ & $246(100 \%)$ & $26(21.0 \%)$ & $98(79.0 \%)$ & $124(100 \%)$ \\
\hline
\end{tabular}

Table 16.

Cerebrospinal fluid leak versus meningitis 


\begin{tabular}{llll|lll}
\hline \multirow{3}{*}{$\begin{array}{l}\text { Timing of } \\
\text { Surgery }\end{array}$} & \multicolumn{5}{c}{ Meningitis } \\
\cline { 2 - 6 } & Yes & No & Total & Yes & No & Total \\
\hline Elective & $4(5.4 \%)$ & $70(94.6 \%)$ & $74(100 \%)$ & $06(66.7 \%)$ & $03(33.3 \%)$ & $09(100 \%)$ \\
Emergency & $4(26.7 \%)$ & $11(73.3 \%)$ & $15(100 \%)$ & $02(18.2 \%)$ & $09(81.8 \%)$ & $11(100 \%)$ \\
Total & $8(9.0 \%)$ & $81(91.0 \%)$ & $89(100 \%)$ & $08(40.0 \%)$ & $12(60.0 \%)$ & $20(100 \%)$ \\
\hline \multicolumn{5}{c}{$\begin{array}{lllll}\text { (1) Chi-square (Yates) }=4.54 ; \mathrm{p}=0.033 \\
\text { (2) Fisher's Exact Test: } \mathrm{p}=0.065\end{array}$} \\
*Cited (29)
\end{tabular}

Table 17.

Timing of surgery versus meningitis

During 1981-1998, among the 211 survivors, 191 (90.5\%) showed no change in their neurologic condition by the last follow-up when compared with admission, 18 (8.6\%) improved, and 2 $(0.9 \%)$ deteriorated. At the second period, there was an increase in neurological improvement (33\%), an increase of deteriorated (15\%) and the remaining $52 \%$ showed no change (Table 18). There was no significant differences between the cauda equina injury and spinal cord (Table 19).

\begin{tabular}{lrr}
\hline & \multicolumn{2}{c}{ Patients } \\
\cline { 2 - 3 } & \multicolumn{1}{c}{$1981-1998^{*}$} & $2005-2008$ \\
\hline Unchanged & $191(90.5 \%)$ & $66(52 \%)$ \\
Improved & $18(8.6 \%)$ & $42(33 \%)$ \\
Worse & $2(0.9 \%)$ & $19(15 \%)$ \\
Total & $211 *(100 \%)$ & $127(100 \%)$ \\
\hline *Cited (29) & \multicolumn{2}{c}{ Comparative Chi-square: $67,45 \mathrm{p}<0.00001$}
\end{tabular}

Table 18.

Outcome

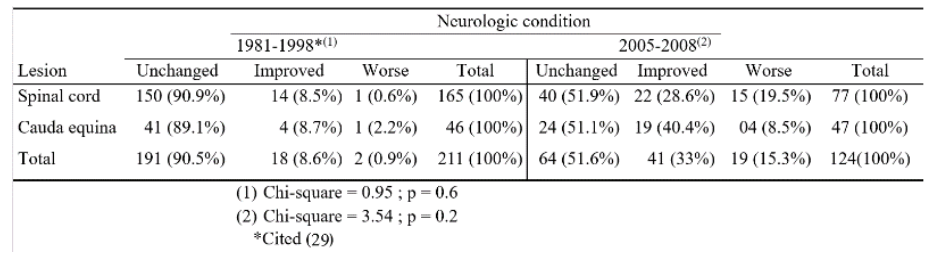

Table 19.

Lesion versus Outcome
Although during the first period patients with metallic fragments had an improvement rate of $13.1 \%$, compared with $3.1 \%$ for those presenting with the full mass of the bullet inside the spinal canal, this difference was not significant during 2005-2008 (Table 20)

\begin{tabular}{|c|c|c|c|c|c|c|c|c|}
\hline \multirow{3}{*}{$\begin{array}{l}\text { Inside canal } \\
\text { Radiologic } \\
\text { Findings }\end{array}$} & \multicolumn{8}{|c|}{ Neurologic condition } \\
\hline & \multicolumn{3}{|c|}{$1981-1998^{*(1)}$} & \multicolumn{5}{|c|}{$2005-2008^{(2)}$} \\
\hline & Unchanged & Improved & Worse & Total & Unchanged & Improved & Worse & Total \\
\hline Bullet & $93(94.9 \%)$ & $3(3.1 \%)$ & $2(2.0 \%)$ & $98(100 \%)$ & $40(51.9 \%)$ & $22(28.6 \%)$ & $15(19.5 \%)$ & $77(100 \%)$ \\
\hline Fragments & $98(86.7 \%)$ & $15(13.3 \%)$ & $0(0.0 \%)$ & $113(100 \%)$ & $24(51.1 \%)$ & $19(40.4 \%)$ & $04(8.5 \%)$ & $47(100 \%)$ \\
\hline Total & $191(90.5 \%)$ & $18(8.6 \%)$ & $2(0.9 \%)$ & $211(100 \%)$ & $64(51.6 \%)$ & $41(33.1 \%)$ & $19(15.3 \%)$ & $124(100 \%)$ \\
\hline
\end{tabular}

Table 20.

Radiologic findings versus outcome

Among those who underwent surgery in both periods, only $12.6 \%$ and $25 \%$ improved but not significantly, keeping neurological unchanged the remaining $87.4 \%$ and $75 \%$ respectively. In the group whose management was conservative, 6 and $40.9 \%$ improved and 93.7 and $59.1 \%$ showed no significant changes (Table 21). Emergency operated patients or elective continued without statistical impact on the outcome (Table 22).

\begin{tabular}{lrrr|rrr}
\hline & \multicolumn{5}{c}{ Outcome } \\
\cline { 2 - 5 } Surgery & Unchanged & \multicolumn{1}{c}{ Improved } & \multicolumn{1}{c}{ Total } & Unchanged & Improved & \multicolumn{1}{c}{ Total } \\
\hline Yes & $76(87.4 \%)$ & $11(12.6 \%)$ & $87(100 \%)$ & $09(75.0 \%)$ & $03(25.0 \%)$ & $12(100 \%)$ \\
No & $119(93.7 \%)$ & $8(6.3 \%)$ & $127(100 \%)$ & $55(59.1 \%)$ & $38(40.9 \%)$ & $93(100 \%)$ \\
Total & $195(91.1 \%)$ & $19(8.9 \%)$ & $214(100 \%)$ & $64(61.0 \%)$ & $41(39.0 \%)$ & $105(100 \%)$ \\
\hline \multicolumn{6}{c}{ (1) Chi-square $=2.57 \mathrm{p}=0.11$} \\
(2) Chi- square (Yates) $=0.56 ; \mathrm{p}: 0.456$ & & \\
& *Cited (29)
\end{tabular}

Table 21.

Surgery versus outcome 


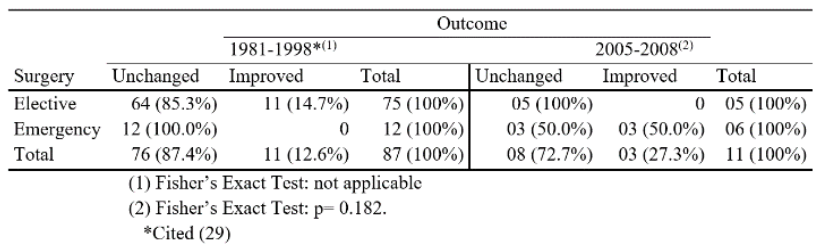

Table 22.

Timing of surgery versus outcome

Laminectomy in patients with cauda equina injury showed no significant changes in any of the two periods. 93.7 and $75 \%$ did not change their neurological status after surgery, $15.4 \%$ improved with conservative management compared to $6.3 \%$, while only $25 \%$ improved after surgery in the second period (table 23).

\begin{tabular}{|c|c|c|c|c|c|c|}
\hline \multirow[b]{3}{*}{ Laminectomy } & \multicolumn{6}{|c|}{ Outcome } \\
\hline & \multicolumn{3}{|c|}{$1981-1998^{*(1)}$} & \multicolumn{3}{|c|}{$2005-2008^{(2)}$} \\
\hline & Unchanged & Improved & Total & Unchanged & Improved & Total \\
\hline Yes & $30(93.7 \%)$ & $2(6.3 \%)$ & $32(100 \%)$ & $03(75.0 \%)$ & $01(25.0 \%)$ & $04(100 \%)$ \\
\hline No & $11(84.6 \%)$ & $2(15.4 \%)$ & $13(100 \%)$ & $02(100 \%)$ & 0 & $02(100 \%)$ \\
\hline Total & 41 (91.1\%) & $4(8.9 \%)$ & $45(100 \%)$ & $05(83.3 \%)$ & $01(16.7 \%)$ & $06(100 \%)$ \\
\hline
\end{tabular}

Table 23.

Cauda Equina Lesions: Laminectomy versus Outcome

When discriminating the role of laminectomy over spinal injuries, it was observed that they had not statistically improved the neurological status of patients, only $10.5 \% \mathrm{did}$, and the remaining $5.3 \%$ improved spontaneously. In the second period, $2(66.7 \%)$ patients improved but the statistical tests were not applicable (Table 24).

\begin{tabular}{|c|c|c|c|c|c|c|}
\hline \multirow[b]{3}{*}{ Laminectomy } & \multicolumn{6}{|c|}{ Outcome } \\
\hline & \multicolumn{3}{|c|}{$1981-1998^{*(1)}$} & \multicolumn{3}{|c|}{$2005-2008^{(2)}$} \\
\hline & Unchanged & Improved & Total & Unchanged & Improved & Total \\
\hline Yes & $34(89.5 \%)$ & $4(10.5 \%)$ & $38(100 \%)$ & $01(33.3 \%)$ & $02(66.7 \%)$ & $03(100 \%)$ \\
\hline No & $108(94.7 \%)$ & $6(5.3 \%)$ & $114(100 \%)$ & $02(100.0 \%)$ & - & $02(100 \%)$ \\
\hline Total & $142(93.4 \%)$ & $10(6.6 \%)$ & $152(100 \%)$ & $03(60.0 \%)$ & $02(40.0 \%)$ & $05(100 \%)$ \\
\hline
\end{tabular}

Table 24.

Spinal cord lesions: laminectomy vs outcome

\section{Discussion}

Yashon et al., believed that an average neurosurgeon would treat no more than two patients with GW to the spine each year [25]. This number is obviously too low to allow a neurosurgeon, working alone, to establish reliable management guidelines. Such guidelines can be drawn only from the analysis of large series, and papers reporting a large number of patients are scarce. Simpson et al., published in 1989 their data on the treatment of 142 patients with this type of injury [20]. Cybuslki et al., described their experience with 88 patients, collected during a period of 19 years [34]. Velmahos and Demetriades in Johannesburg, published a series of 153 patients with GWS [35].

Levy et al., in Los Angeles, studied 252 patients treated between 1980 and 1993 [36]. Also from the United States came the publication by Heary et al., who reported 254 patients treated during a period of 15 years [37]. Azevedo- Filho et al., [26] published in 2001 one of the most extensive cohorts in the neurosurgical literature, the study of 246 patients in the period 1981-1998.

Although retrospective, its size and thorough follow-up permitted to throw 
some light on the most important aspects of this pathology, specifically the response to surgical treatment. In this new paper the authors compare that study of 246 patients conducted from 1981 to 1998 with a new cohort of 127 patients studied ten years later during the period 2005-2008. Table 1 shows that in the last three years only $50 \%(\mathrm{~N}=127)$ of patients were admitted compared to the first period, which shows the true pandemic of violence affecting most urban centers around the world during the first period (Table 1). In civilian $\mathrm{GW}$, men are more likely to be the victims: several authors reported rates around 94\% [33, 34, 35, 36, 37, 38, 39]. The mean age of the patients varied from 25 to 32 years $[24,34]$. The present study showed a male incidence of $95.5 \%$ and $85.8 \%$ and the mean age of patients was 26.6 years and 25 years respectively.

The neurologic status at admission attests the high complication rate of this pathology; $62.2 \%$ and $69.3 \%$ respectively, did not have motor function at admission (see Table 2). The period 2005-2008 showed a significant increase in patients classified as Frankel B previously considered A. The authors take this as a reporting bias (misclassification) at the first paper due to an error of the examiner to assess the sensitivity of the patient. Many factors seem to work together to make guns so destructive to neural tissues.

Yashon et al., pointed to the cavitation effect and shock waves as having considerable destructive power, even when the missile has not violated the spinal canal [40]. The velocity of the missile is more important than its mass as a determinant of destructive properties. Velocity is directly related to the generation of shock waves [41]. According to Benzel et al., because civilian missiles have a lower velocity than military ones, the destructive effects are more related to direct injury of neural tissues [26]. The precise follow-up period needed for conclusions to be drawn is a subject of controversy in the literature. Guttmann believed that patients who have complete lesions for more than 2 weeks probably would remain unchanged for life [2]. Yashon et al., considered a period of 6 months of observation as a landmark to exclude the possibility of functional improvement [40]. Hammoud et al., studying 64 patients, based their conclusions on a mean follow-up of 10 weeks [13]. In this study the mean follow-up in both periods was 76.3 days which was 
considered long enough to validate conclusions.

The death rate observed in this study was $14.2 \%$ and $12.6 \%$ (see Tabla 3). Cushing, during World War I, reported a death rate of $71 \%$ [42]. Guttmann, 40 years later, reduced the death rate to less than $10 \%$ [4]. Heiden et al., and Benzel et al., working in the United States and reporting injuries of civilians, also found a death rate of around $10 \%[26,27]$. However, the absence of death in several publications from the United States [19, $34,43,44]$ reflects the excellence of medical care in those centers. Thus, a death rate of $14.2 \%$ and $12.6 \%$ is common for most series.

Tables 5, 6, and 7 emphasize the role played by respiratory infections and its association with death. Absence of motor power and cervical injuries are significantly associated with pulmonary infections, Frankel A and B had about three times more risk of pulmonary infection in this paper. Therefore, any patient arriving at the hospital in poor neurological condition and who has a high lesion should immediately undergo a vigorous regimen of respiratory physiotherapy. Kupcha et al., analyzing patients with cervical wounds, found a $42.9 \%$ rate of pulmonary infections, similar to the one found in this review (48.6\% and 44.4\%) [24].

Table 7 shows that $41.4 \%$ of the patients with pulmonary infections eventually died, in contrast to the population without this kind of infection, in which only $5.9 \%$ died. These differences were significant, once again stressing the importance of preventing this type of complication. Therefore, in the second period a fall of this complication is achieved (10.5\%) at statistically not significant levels due to diagnosis and early aggressive treatment of the same. Guttmann although a leader in the conservative care philosophy, recommends surgical treatment for patients with GW to the spine as soon as possible [4]. Yashon et al., performed DL in $69.2 \%$ of their patients [40]. Stauffer et al., reported that $54.6 \%$ of patients were surgically treated; Robertson and Simpson reporting cauda equina lesions, operated on around 50\% of their patients [19, 44]. However, Benzel et al., Simpson et al., and Kupcha et al., reported lower percentages of patients undergoing surgery (respectively, 37.1\%, 21.8\%, and $17.8 \%$ ) [24, 26, 39]. Table 8 shows a significant increase towards a more conservative management $(36.2 \%$ to 
$20 \%$ ), in accord with the overall tendency shown in the literature.

Incomplete lesions (grades B, C, D) seem to play a role in persuading the surgeon to suggest an operative procedure. Benzel et al., operated on $15 \%$ of their grade A patients but $66.7 \%$ of the patients with incomplete lesions [26]. This tendency was objectified at the first period in this report (undergo surgery to $60.9 \%$ ), but in the second period there was a significant tendency to statistically treat grade $B, C, D$ patients conservatively (see Table 9). The differences reached significance at the first period and reflect the long accepted concept that DL would mostly benefit patients who had some neurologic function.

This fact was not reflected in better outcome, determining management change during 2005-2008. Not many publications seem to have paid attention to the timing of surgery. Emergency surgery might have played a role in the outcome of the patients. Yashon et al., operated on $80 \%$ of their patients during the first 24 hours after the trauma, Stauffer et al., on $57.4 \%$. Benzel et al., performed emergency laminectomies in only $7.7 \%$ of their series $[19,26,40]$. At the first period in this report, emergency procedures were performed in $16.9 \%$ of those who underwent surgery, which is certainly not high. Patients with complete loss of function (grade A) underwent more emergency operations than those in grades B, C, and D. The differences were significant (see Table 10) and suggest that, unlike the data in Table 9, complete lesions more frequently drove the neurosurgeon to consider an emergency laminectomy. However, at the second period there was a tendency of significant increase in emergency surgery especially in patients with incomplete lesions due to the best outcome observed (see Table 10). This observation has not received much interest in the literature. Kupcha et al., are among the few authors who analyzed the presence of the full mass of the bullet inside the spinal canal in relation to the timing and likelihood of surgical procedures [24]. Table 11 shows that a bullet inside the spinal canal significantly directed the neurosurgeon toward a surgical option during the first period. Interestingly, the presence of the bullet was not sufficient to prompt an emergency operation (see Table 12). In the second period the presence full mass of the bullet inside the spinal canal there was not significant difference respect to fragments inside spinal canal. 
Anecdotal reports have claimed throughout the decades that patients of the last century with cauda equina injuries would have a better outcome if they underwent surgical treatment. Robertson and Simpson reported that $54.5 \%$ of their 33 patients with cauda equina lesions underwent DL [24].

Table 13 shows that $72.3 \%$ of our patients during 1981-1998 underwent surgery, in comparison with $27.6 \%$ of those with injuries affecting the spinal cord. These differences were relevant. In the second period there were not relevant differences denoting a substantial comparative decline in cauda equina surgery. The presence of CSF leak is relatively common in patients with gunshot wounds to the spine. In this review, we found $10(4.1 \%)$ and 12 (9.7\%) patients respectively with this complication significantly associated with the surgical management. Our analysis shown that operated patients have seven times more risk of CSF leak (see Table 14). The differences were significant and pointed out that DL is a risk factor that patients and surgeons must face in these circumstances.

Stauffer et al., reported, among patients surgically treated, an incidence rate of 5.9\% [19] and, Aarabi et al., in a military experience, reported that almost $15 \%$ of the surgical population had this complication [15]. At the first study period operated patients showed $9 \%$ Meningitis, increasing significantly to $42.9 \%$ in the second period, which meant four times the risk of complication (see Table 15). Simpson et al., are among the few authors who have related CSF leak to meningitis [39]. This review shows a likelihood that $40 \%$ of patients with CSF leak will develop meningitis, with a much lower incidence in those without elimination of the fluid. The differences were of great importance, emphasizing the need for a watertight closure of the dura mater.

Also found an incidence rate of meningitis three times more in patient who underwent surgery versus those who were conservatively managed. Aarabi et al., found an overall incidence of meningitis in $7.3 \%$ of their series and agreed that surgical treatment is a risk factor [15]. Bivarate analysis shows the significant risk of meningitis that surgery can bring, among 16 to 19 times (see Table 16). Emergency surgery was associated only in the first period of study (Table 17).

The differences were significant, pointing to the increased risk of death in patients with complete loss of neurologic function. Most publications 
lack precise methodological parameters for gauging outcome. For example, Cybulski et al., considered a significant neurologic improvement when the sensory level was found to have lowered at least two segments, or when some motor improvement was detected [34]. Using this imprecise methodology, they reported a $41 \%$ rate of neurologic improvement. Stauffer et al., studying 79 patients with incomplete lesions, reported noticeable motor progress in $71 \%$ of the surgically treated group and in $76.5 \%$ of those conservatively managed [19].

We strongly believe that a precise and reproducible way to gauge neurologic improvement or deterioration should take into consideration a modification in neurologic function of at least one grade according to the Frankel/ASIA/IMSOP classification. Aarabi et al., are among the few authors who also ascribe to this idea and use these criteria, reporting no change in $75 \%$ of patients and improvement in only $10 \%$ [15].

Table 18 shows that among the survivors in the first study compared to the second, $90.5 \%$ and $52 \%$ remained unchanged; $8.6 \%$ and $33 \%$ improved; $0.9 \%$ and $15 \%$ worsen, the difference was statistically important. Traditionally, neurosurgical lore suggests that cauda equina injuries have a better prognosis than lesions affecting the spinal cord [40]. Many authors agree that spinal wounds below L1 have a less gloomy outcome [26, 34]. Unfortunately, this was not the message extracted from our data, where patients with spinal cord and cauda equina injuries behaved in a similar way in both periods (see Table 19).

Yashon et al., postulated that the presence of the entire mass of the bullet inside the spinal canal would produce a worse outcome [40]. This has been echoed by Stauffer et al. Our study showed that patients with metallic fragments inside the canal fared statistically better than those with the entire mass of the bullet in the canal at the first period, but it was not statistically significant in 2005-2008 (see Table 20).

The impact of any therapy on the outcome of patients with gunshot wounds to the spine requires a better prospective analysis. Still, based on these data, some generalizations can be made. Yashon et al., postulated that decompressed laminectomies influenced the outcome of their patients; this opinion was shared by Heiden et al., Stauffer et al., and Cybulski et al., [19, 27, 34, 40]. Hammoud et al., stated that at least surgical treatment would offer a 
psychological benefit to the patients and relatives [13]. We disagree with this conclusion, based on our data. Table 21 shows that in the surgical group, $12.2 \%$ improved in 1981-1998 and $25 \%$ in 2005-2008. In the nonsurgical group, $6.3 \%$ got better at the first period and $40.9 \%$ in $2005-2008$. The differences are not statistically relevant. Despite the unfavorable results in the surgical group, neurosurgeons still often advocate DL.

It is even more difficult to support emergency surgery when we found that no patient benefited from this policy (see Table 22). Benzel et al., concluded that DL should not be denied to patients with cauda equina injuries because surgical treatment should play a positive role in the outcome [26]. However, our data showed that conservative management did not have any difference compared with surgery (see Table 23). Thus, we found that decompressed laminectomy could not be significantly associated with a better outcome (see Table 24) at any level. From our data it seems difficult to find a scientifically based reason to recommend surgery to patients with GWS to improve function. We agree with Yashon et al., when they stated that the final result of these spine injured patients is related to their initial neurologic status and entirely unassociated with DL or other surgery [40].

In conclusions, this work allowed us to see how the results observed in the first study determined therapeutic behavior change in the second period and how these changes remained without statistical significance. In fact, we have demonstrated one more time that DL has no positive impact on the outcome of patients with gunshot wounds to the spine and the surgical procedures continued significantly associated with higher rates of CSF leak and meningitis. We no longer recommend surgery for this patients but we strongly believe that a prospective multicenter study should be organized with a larger population of patients to allow a better understanding of the complexity of this pathology.

\section{Competing interests}

Authors declare that we have no competing interests.

\section{Authors Contributions}

All authors wrote, read and approved the final manuscript.

\section{References}

1. Roye WP, Dunn EL, Moody JA. Cervical spinal cord injury - a public catastrophe. Trauma 1988; 28:1260-1264.

2. Farmer JC, Vaccaro AR, Balderston RA, Albert TJ, Cotler J. The changing nature of admission to a spinal cord injury center: violence on the rise. J Spinal Disord 1998; 11:400-403. 
3. Tator $\mathrm{CH}$. Classification of spinal cord injury based on neurological presentation. In: Narayan RK, Wilberger JE, Povlishock JT, eds. Neurotrauma. New York: McGraw-Hill; 1996:10591073.

4. Guttmann L. Spinal cord injuries: comprehensive management and research. 2d ed. Oxford: Blackwell Scientific Publications; 1976.

5. Ducker TB, Lucas JT, Wallace CA. Recovery from spinal cord injury. In: Weiss MH, ed. Clinical neurosurgery. Baltimore: Williams \& Wilkins; 1982:495-513.

6. Ohry A, Ohry-Kossoy K. Spinal cord injuries in the 19th century: background, research and treatment. Edinburgh: Churchill Livingstone; 1989.

7. Sonntag VKH: History of degenerative and traumatic diseases of the spine. In: Greenblat SH, Epstein MH, eds. A history of neurosurgery in its scientific and professional contexts. Park Ridge: American Association of Neurological Surgeons; 1997:355-371.

8. Gobo DJ. Localization techniques: neuroimaging and electroencephalography. In: Greenblat SH, Epstein MH, eds. A history of neurosurgery in its scientific and professional contexts. Park Ridge: American Association of Neurological Surgeons; 1997: 223-446.

9. Cushing H. Haematomyelia from gunshot wounds to the cervical spine. Acta Chir Scand 1897; 8:195-197.

10. Cushing H. Haematomyelia from gunshot wounds to the spine: a report of two cases with recovery following symptoms of hemilesion of the cord. Am J Med Sci 1898; 115:654-683.

11. Matson DM. The treatment of acute compound injuries of the spinal cord due to missiles. Springfield, IL: Charles Thomas; 1948.
12. Spurling RG: The European Theater of operations. In: Woodhall B, ed. Neurosurgery. Washington: US Government Printing Office; 1959.

13. Hammoud MA, Haddad FS, Moufarrij NA. Spinal cord missile injuries during the Lebanese civil war. Surg Neurol 1985; 43:432-442.

14. Ohry A, Rozin R. Acute spinal cord injuries in the Lebanon war. Israel J Med Sci 1984; 20:345-349.

15. Aaarabi B, Alibaii E, Taghipur M, Kamgarpur A. Comparative study of functional recovery for surgically explored and conservatively managed spinal cord missile injuries. Neurosurgery 1996; 39:1133-1140.

16. Splavski B, Vranković D, Sarić G, et al. Early management of war missile spine and spinal cord injuries: experience with 21 cases. Injury 1996; 27:699-702.

17. Six E, Alexander E, Kelly DL. Gunshot wounds to the spinal cord. South Med J 1979; 72:699-702.

18. Miller CA. Penetrating wounds of the spine. In: Wilkins RH, Rengashary SS, eds. Neurosurgery. Vol. 1. San Francisco: McGraw- Hill; 1985:1746-8.

19. Stauffer ES, Wood RW, Kelly EG. Gunshot wounds of the spine: effects of laminectomy. J Bone Joint Surg Am 1979; 61:389-392.

20. Simpson RK, Venger BH, Narayan RK. Penetrating spinal cord injury in a civilian population: a retrospective analysis. Surg Forum 1986; 37:494-496.

21. Jacobson AS, Borg E. Spinal cord injury in vietnamese combat. Paraplegia 1970; 7:263-281.

22. Meirowsky AM. Penetrating spinal cord injuries. In: Coates J, Meirowsky AM, eds. Neurological surgery of trauma. Washington: US Government Printing Office; 1965:257-344.

23. Demetriades D, Theodorou D, Cornwell $\mathrm{E}$, et al. Transcervical gunshot injuries: 
mandatory operation is not necessary. $J$ Trauma 1996; 40:758-760.

24. Kupcha PC, An HS, Cotler JM. Gunshot wounds to the cervical spine. Spine 1990; 15:1058-1063.

25. Yashon D. Spinal injury. New York: Appleton; 1978.

26. Benzel EC, Hadden TA, Coleman JE. Civilian gunshot wounds to the spinal cord and cauda equina. Neurosurgery 1987; 20:281-285.

27. Heiden JS, Weiss MH, Rosenberg AW, Kurze T, Apuzzo ML. Penetrating gunshot wounds of the cervical spine in civilians. J Neurosurg 1975; 42:575-579.

28. Yoshida GM, Garland D, Waters RL. Gunshot wounds to the spine. Orthop Clin North Am 1995; 26:109-116.

29. Azevedo-Filho H, Martins C, CarneiroFilho G, et al. Gunshot Wounds to the Spine: Study of 246 Patients. Neurosurgery Quarterly 2001;11:199208.

30. Bailey RW. Fractures and dislocations of the cervical spine. In: Cervical Spine Research Society, eds. The cervical spine. Philadelphia: Lea and Febiger; 1974:116-145.

31. White AA, Johnson RM, Panjabi MM, Southwick WO: Biomechanical analysis of clinical spine stability in the cervical spine. Clin Orthop 1975; 109:85-95.

32. Denis F. The three column spine and its significance in the classification of acute toracolumbar spinal injuries. Spine 1983; 8:817-831.

33. Roy-Camille R, Saillant G, Mazel C. Internal fixation of the unstable cervical spine by posterior osteosynthesis with plates and screws. In: Cervical Spine Research Society, eds. The cervical spine. 2d ed. Philadelphia: Lippincott; 1989:390-403.

34. Cybulski GR, Stone JL, Kant R. Outcome of laminectomy for civilian gunshot injuries of the terminal spinal cord and cauda equina: review of 88 cases. Neurosurgery 1989; 24:392-397.

35. Velmahos G, Demetriades D. Gunshot wounds to the spine: should retained bullets be removed to prevent infection? Ann R Coll Surg Engl 1994; 76:85-87.

36. Levy ML, Gans W, Wijesinghe HS, et al. Use of methylprednisolone as an adjunct in the management of patients with penetrating spinal cord injury: outcome analysis. Neurosurgery 1996; 39: 1141-1149.

37. Heary RF, Vaccaro AR, Mesa JJ, et al. Steroids and gunshot wounds to the spine. Neurosurgery 1997; 41:576-584.

38. Mckinley W, Cifu D, Keyser ML, Wilson K. Comparison of rehabilitation outcomes in violent versus non-violent traumatic spinal cord injury. J Spinal Cord Med 1998; 21:32-36.

39. Simpson RK, Venger BH, Narayan RK. Treatment of acute penetrating injuries of the spine: a retrospective analysis. $J$ Trauma 1989; 29:42-46.

40. Yashon D, Jane DA, White RJ. Prognosis and management of spinal cord and cauda equina bullet injuries in sixty-five civilians. J Neurosurg 1970; 32:163-170.

41. Demuth WE. Bullet velocity as determinants of wound capability: an experimental study. J Trauma 1966; 6:222-232.

42. Harvey Cushing Society: A bibliography of the writings of Harvey Cushing. 3d ed. Park Ridge: American Association of Neurological Surgeons; 1993.

43. Frankel HL, Hancock DO, Hyslop G, et al. The value of the postural reduction in the initial management of closed injuries of the spine with paraplegia and tetraplegia. Spine 1969; 7:179-192.

44. Robertson DP, Simpson RK. Penetrating injuries restricted to the cauda equina: a retrospective review. Neurosurgery 1996; 31:265-270. 